\title{
Taming effect of PCPA and 5-HTP in septal rats
}

\author{
ROBERT F. SMITH \\ George Mason University, Fairfax, Virginia
}

\begin{abstract}
Previous authors have separately reported that the hyperreactivity syndrome commonly found after septal lesion can be attenuated by injection of either a serotonin depletor, PCPA, or a serotonin precursor, 5-HTP. The present experiment used a factorial design to investigate these apparently discrepant results. As previously reported, each drug produced a taming effect in septal animals, and additionally, the combination of the two drugs tended to have an additive, rather than an antagonistic, effect. This suggests that the two drugs might share a common neurochemical action. Since Sloviter, Drust, and Connor (1978) have reported that PCPA has an early serotonin-releasing effect, it appears that serotonin release, not depletion, has a taming effect in septal animals.
\end{abstract}

Brady and Nauta (1953) and a large number of subsequent authors have described a syndrome of hyperreactivity following septal lesion. Although factors such as pre- and postoperative handling have been found to ameliorate the hyperreactivity (Nielson, McIver, \& Boswell, 1965; Seggie, 1968), the anatomical and biochemical bases of the syndrome have not been well defined. Three neurochemical systems have been implicated in septal-lesion-induced hyperreactivity. Stark and Henderson (1972) reported that physostigmine injections diminished the hyperreactivity, suggesting a cholinergic component to the syndrome. Unfortunately, because they did not report activity scores in unoperated subjects, it is not possible to determine whether their injections returned reactivity to normal; it appears that there was only a slight reduction in reactivity. Several authors have reported that dopamine (DA) agonists reduce hyperreactivity (Olton \& Gage, 1974; Marotta, Logan, Potegal, Glusman, \& Gardner, 1977), and that such reduction appears to be essentially permanent. Some of the doses used in those studies were very high, and there has been no indication of the mechanism by which short-acting DA agonists might produce long-term alleviation of septal-lesion-induced hyperreactivity.

A third neurotransmitter system has been implicated by apparently conflicting reports. Dominguez and Longo $(1969,1970)$ reported that injection of parachlorophenylalanine (PCPA-a serotonin depletor) in septal-lesioned animals resulted in an immediate reduction of reactivity, suggesting that serotonin depletion reduces reactivity in septal-lesioned animals. Smith (1979b), noting that septal lesions themselves reduce forebrain serotonin (Lints \&

Requests for reprints should be addressed to the author at: Department of Psychology, George Mason University, 4400 University Drive, Fairfax, Virginia 22030.
Harvey, 1967), found that repleting forebrain serotonin to nearly normal by injection of $105 \mathrm{mg} / \mathrm{kg}$ of d,1-5-hydroxytryptophan (5-HTP) reduced footshock sensitivity and general reactivity of septal animals to nearly normal. Since PCPA depletes serotonin, whereas 5-HTP increases serotonin, it is clear that the reports of Dominguez and Longo (1970) and Smith (1979b) are apparently discrepant. It is difficult to understand how two drugs with reported opposite effects can have similar actions on septal animals. There are at least two possible explanations for the apparent discrepancy: (1) the discrepancy may be an artifact of subtle differences in experimental procedures; (2) the common behavioral effect of 5-HTP and PCPA may be real, which would therefore suggest that the two drugs share some common (neurochemical?) action. The latter possibility exists despite the reported serotonin-depleting effects of PCPA. The depletion effects are maximal at 2-3 days postinjection, while the calming effects reported by Dominguez and Longo were present at $30 \mathrm{~min}$ postinjection.

The present study was designed to behaviorally assess the actions of 5-HTP and PCPA on septal animals in a single study. If PCPA and 5-HTP have opposite neurochemical actions at the time of testing, one would expect that they would have different behavioral effects on septal animals and also that the effects of one would antagonize the effects of the other. The present study was designed to determine whether a common effect on behavior exists, and whether the two drugs have an antagonistic effect on each other.

\section{METHOD}

\footnotetext{
Subjects

The subjects were naive male Sprague-Dawley rats, weighing $250-300 \mathrm{~g}$ at the time of surgery. All subjects were housed singly
} 
with ad-lib access to food and water, and were maintained on a 12:12-h light-dark cycle. All subjects received septal lesions.

\section{Surgical Procedures}

With the rat under $45-\mathrm{mg} / \mathrm{kg}$ sodium pentobarbital anesthesia, the scalp was incised, burr holes were drilled in the skull, and and insulated electrode ( 00 insect pin, Scotchkote insulation, $.5-\mathrm{mm}$ exposed tip) was stereotaxically positioned to $1.0 \mathrm{~mm}$ anterior to bregma, $.5 \mathrm{~mm}$ lateral to midline, and $5.0 \mathrm{~mm}$ below dorsal skull surface. Bilateral electrolytic lesions were effected by passage of a $2.5-\mathrm{mA}$ cathodal current for $30 \mathrm{sec}$. The subjects were allowed to recover for 7 days prior to testing.

\section{Injection Protocols}

PCPA. Parachlorophenylalanine methyl ester hydrochloride (PCPA) (Regis Chemical Co., Morton Grove, Illinois) was administered in a $50-\mathrm{mg} / \mathrm{ml}$ solution and a $100-\mathrm{mg} / \mathrm{kg}$ dosage. Dominguez and Longo (1969) reported that $100 \mathrm{mg} / \mathrm{kg}$ of PCPA produced a small but significant calming effect in septal animals. Solutions were freshly mixed immediately prior to injection. The injection control for PCPA was an equal volume of physiological saline.

5-HTP. The agent d,1-5-hydroxytryptophan (5-HTP) (Regis Chemical Co., Morton Grove, Illinois) was administered in a $10-\mathrm{mg} / \mathrm{ml}$ solution, at a dosage of $50 \mathrm{mg} / \mathrm{kg}$. Smith $(1979 \mathrm{~b})$ found that $105 \mathrm{mg} / \mathrm{kg}$ of 5 -HTP had a marked calming effect; therefore, to avoid a ceiling effect, a smaller dose was selected for the present study. Solutions were freshly mixed immediately prior to injection. The injection control for 5-HTP was an equal volume of physiological saline.

\section{Procedure}

All reactivity evaluations were performed using Seggie's (1968) scale, which evaluates magnitude of reactivity to pencil tap (0-4), number of pencil taps to habituation (0-20), and magnitude of reactivity to capture $(0-4)$. On the day prior to testing, all subjects were evaluated for reactivity to capture. The subjects that did not score at least 3 on a scale of $1-4$ were excluded from the study.

On the testing day, an assistant administered all injections; the assistant retained the records of injection until after testing. Thus, the evaluator was unaware of the injection history of each animal. In a $2 \times 2$ design, each subject received either PCPA or saline, and either 5-HTP or saline. Each subject received two injections in the various paired combinations. One hour after injection, the subject was evaluated for reactivity to the three items on the scale. One week following testing, the subjects were sacrificed by overdose of sodium pentobarbital, perfused intracardially with saline followed by $10 \%$ formol-saline, and sectioned for histological examination.

\section{RESULTS}

\section{Histology}

The lesions were assessed without reference to drug condition or reactivity ratings of each animal. Several animals were discarded for caudate damage and/ or substantial neocortical damage, leaving the following group sizes: saline-8, 5-HTP-9, PCPA12, 5-HTP + PCPA-9. Lesions of animals included in data analysis were large and bilaterally symmetrical. Medial and lateral septal areas were substantially destroyed, with consistent ancillary damage to the nucleus accumbens septi, the body of the fornix, and the overlying corpus callosum.

\section{Behavior}

The data for the three behavioral measures were analyzed independently using $2 \times 2$ factorial analyses of variance. For the reactivity scores (to capture and to pencil tap), nonparametric statistics would ordinarily be more appropriate. However, if 5-HTP and PCPA are not antagonistic, a major prediction is that there would be a lack of an interaction effect between the two drugs, and the more powerful analysis of variance was selected to determine if such an interaction occurred. In addition to individual ratings, a global reactivity rating was constructed by combining the three individual ratings. This was done by multiplying the reactivity scores by 5 (so that each measure was on a 0-20-point scale) and then summing all three measures, to give an overall rating between 0 and 60 .

The data for individual behavioral assessments indicated that there was a significant main effect of 5 -HTP on each measure (ps $<.05$ ). In each analysis, the reactivity measure was lower in the 5-HTP condition than in the saline condition. While there was a tendency for PCPA animals to be less reactive than controls, this trend reached significance only in the case of reactivity to pencil tap.

The global reactivity measure (see Figure 1) dem-

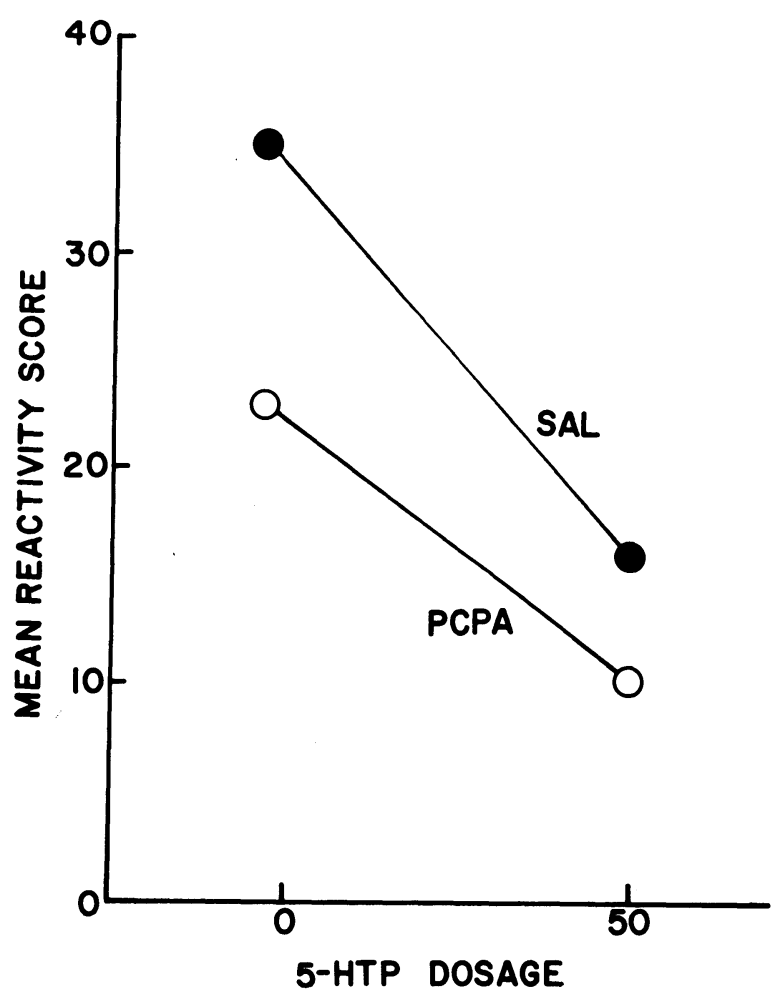

Figure 1. Reactivity scores. The global reactivity scores in the figure were constructed by combining each of the three indlvidual scores. Note the lack of interaction between 5-HTP and PCPA. 
onstrated a significant main effect of 5-HTP $[\mathrm{F}(1,34)$ $=15.48, \mathrm{p}<.001]$, as well as a significant main effect of PCPA $[F(1,34)=5.07, p<.05]$. There were no significant interaction effects on any measure. There was a trend toward interaction in the reactivityto-pencil-tap measure, but this did not reflect any antagonism between PCPA and 5-HTP; rather, it appears that there was a floor effect on this measure. These data provide no suggestion of any antagonism between PCPA and 5-HTP. Rather, the effects of $100 \mathrm{mg} / \mathrm{kg}$ of PCPA are similar to, but weaker than, the effects of $50 \mathrm{mg} / \mathrm{kg}$ of 5 -HTP on the measures reported here.

\section{DISCUSSION}

The most important finding of the present paper is that PCPA and 5-HTP have similar calming effects on septal subjects, and that previous separate reports of the effects of these drugs are not artifacts of differences in experimental procedures. The present data demonstrate that the effects of PCPA and 5-HTP tend to be additive, rather than antagonistic, at $1 \mathrm{~h}$ postinjection. This strongly suggests that the calming effects of PCPA and 5-HTP are mediated through similar (or at least not opposite) mechanisms. Since PCPA's reported effect is depletion of serotonin, while 5-HTP increases serotonin, these data require some explanation. One explanation with which the present data are consistent is that the early postinjection effects of PCPA are different from the depletion seen at 2-3 days postinjection. In particular, it is plausible that the early postinjection effects of PCPA may include presynaptic release of serotonin. Sloviter, Drust, and Connor (1978) have recently shown that PCPA has an early postinjection effect that resembles stimulation of postsynaptic serotonin receptors. They suggested that the effect is produced by displacement of presynaptic serotonin by parachlorophenylethylamine, a metabolite of PCPA, and consquent enhanced release of serotonin. While the data of the present paper provide little insight into the precise mechanism of action, they are consistent with the notion that PCPA has an early postinjection effect similar to that produced by increasing serotonin levels with 5-HTP injection. The data of Sloviter et al. (1978) appear to resolve the apparent inconsistency of both 5-HTP's and PCPA's producing calming effects in septal animals, as reported here. The calming effects of PCPA immediately postinjection appear to be mediated, not by the depletion of serotonin reported 2-3 days postinjection, but by an early action of increased presynaptic release.

One study has reported that PCPA treatment for 5 days prior to septal lesion reduces the magnitude of the hyperreactivity syndrome (Harrell \& Balagura, 1975). Those data are difficult to reconcile with the notion that serotonin depletion contributes to the hyperreactivity syndrome. One possible explanation is suggested by the pattern of Harrell and Balagura's results. They reported that PCPA given for only 2 days prior to lesion did not ameliorate the syndrome, although serotonin depletion from the injections should have been well developed at the time of lesion. Administration of PCPA produces a hyperreactivity syndrome, although one not as pronounced as that produced by septal lesion (Smith, 1979b). It is possible that Harrell and Balagura's subjects injected for the 5 days prior to surgery had developed a hyperreactivity syndrome due to serotonin depletion, had partly habituated to it (diminution of the syndrome is seen with time and handling), and showed only slight symptoms of additional hyperreactivity due to the subsequent lesion. The subjects that received PCPA for only 2 days prior to lesion would have developed significant serotonin depletion only near the time of lesion, and would therefore have had insufficient time for the syndrome produced by PCPA to diminish before lesion. Since Harrell and Balagure did not assess reactivity in their PCPA subjects prior to surgery, this scenario cannot be effectively evaluated for their subjects, but their data do not necessarily invalidate the conclusions presented here.

Although not tested in the present study, it appears that both drugs have calming effects only in hyperreactive subjects. Smith (1979b) reported that larger doses of these drugs $(105 \mathrm{mg} / \mathrm{kg}$ of 5 -HTP and $300 \mathrm{mg} / \mathrm{kg}$ of PCPA) had nonsignificant effects on the behavior of unlesioned animals, as assessed by the same technique used in the present study.

The present data reinforce the notion that a component of septal hyperreactivity is due to serotonin depletion produced by the lesion (Smith, 1979a, 1979b). This hypothesis was apparently contradicted by the calming effects of PCPA. If PCPA produces an early postinjection release of serotonin, the immediate calming effects of PCPA in septal animals support, rather than refute, the notion that increased serotonin partly reverses the hyperreactivity produced by septal lesion. The apparent involvement of serotonin depletion in the septal syndrome does not, however, exclude the participation of other neurotransmitters. Since no single manipulation has been demonstrated to return septal animals completely to normal, it may be that no single change produced by the lesion is responsible for the hyperreactivity. The recent finding by Chafetz, Thompson, Evans, and Gage (1981) that the hyperreactivity syndrome cannot be mimicked by neurotoxic lesions that deplete individual transmitters supports this position. Work to date thus suggests that the hyperreactivity syndrome may be due to an interaction of depletion of several transmitters; the specific pattern of depletion required may be specified by further work. 


\section{REFERENCES}

Brady, J. V., \& Nauta, W. J. H. Subcortical mechanisms in emotional behavior: Affective changes following septal forebrain lesions in the albino rat. Journal of Comparative and Physiological Psychology, 1953, 46, 339-346.

Chafetz, M. D., Thompson, R. G., Evans, S. H., \& Gage, F. H. Biochemical specificity of septal hyperreactivity: $A$ behavioral discrimination. Behavioural Brain Research, 1981, 2, 409-420.

Dominguez, M., \& Longo, V. G. Taming effects of parachlorophenylalanine on septal rats. Physiology \& Behavior, 1969, 4, 29-31.

Dominguez, M., \& Longo, V. G. Effects of p-chlorophenylalanine, a-methylparatyrosine, and of other indol- and catecholamine depletors on the hyperirritability syndrome of septal rats. Physiology \& Behavior, 1970, 5, 607-610.

Harrell, L. E., \& Balagura, S. Septal rage: Mitigation by pre-surgical treatment with p-chlorophenylalanine. Pharmacology, Biochemistry, and Behavior, 1975, 3, 157-159.

Lints, C. E., \& Harvey, J. A. Altered sensitivity to electric shock and decreased brain content of serotonin following brain lesions in the rat. Journal of Comparative and Physiological Psychology, 1967, 67, 23-31.

Marotta, R. F., Logan, N., Potegal, M., Glusman, M., \& GARDNER, E. L. Dopamine agonists induce recovery from surgically-induced septal rage. Nature, 1977, 269, 513-515.
Nielson, H., McIver, H., \& Boswell, R. Effects of septal lesions on learning, emotionality, activity, and exploratory behavior in rats. Experimental Neurology, 1965, 11, 147-157.

Olton, D. S., \& GAGE, F. H. Behavioral, anatomical, and biochemical aspects of septal hyperreactivity. In J. F. DeFrance (Ed.), The septal nuclei. New York: Plenum Press, 1974.

SEGGIE, J. Effect of somatosensory stimulation on affective behavior of septal rats. Journal of Comparative and Physiological Psychology, 1968, 66, 820-822.

Sloviter, R. S., Drust, E. G., \& Connor, J. D. Serotonin agonist actions of p-chlorophenylalanine. Neuropharmacology, 1978, 17, 1029-1033.

SмiтH, R. F. Attenuation of septal lesion-induced shuttlebox facilitation by S-hydroxytryptophan. Physiological Psychology, 1979, 7, 419-421. (a)

Smith, R. F. Mediation of footshock sensitivity by serotonergic projection to hippocampus. Pharmacology, Biochemistry, and Behavior, 1979, 10, 381-388. (b)

Stark, P., \& Henderson, J. K. Central cholinergic suppression of hyperreactivity and aggression in septal-lesioned rats. Neuropharmacology, 1972, 11, 839-847.

(Manuscript received April 5, 1982; revision accepted for publication July 25, 1982.) 